\title{
Development and Production of Prototype Model of Axial Fan
}

Josef Sedlak $^{1}$, Jiri Malasek ${ }^{2}$, Martin Ondra ${ }^{2}$, Ales Polzer ${ }^{1}$

${ }^{1}$ Department of Machining Technology, Institute of Manufacturing Technology, Faculty of Mechanical Engineering, Brno University of Technology, Technická 2896/2, Brno 616 69, Czech Republic. E-mail: sedlak@fme.vutbr.cz, polzer@fme.vutbr.cz

${ }^{2}$ Department of Handling and Building Machines, Institute of Automotive Engineering, Faculty of Mechanical Engineering, Brno University of Technology, Technická 2896/2, Brno 616 69, Czech Republic. E-mail: malasek@fme.vutbr.cz, m.rtin@seznam.cz

Additive technologies belong to modern trends in production of prototype components. They include e.g. sintering of powders with a various chemical composition, granularity, physical, chemical and other usable qualities. There belong to additive technologies especially a technology of Rapid Prototyping that use various principles to make components. According to the used technology photopolymers, thermoplastics, a specially modified paper or metal powders are used in a Rapid Prototyping machinery.

An article deals with a characteristic, concept, calculation and production of a prototype model of an axial fan that enables quick build-up time of fan turbine speed. Production of the axial fan is realized by an additive technology of Rapid Prototyping using a method of Fused Deposition Modelling - a 3D printer Fortus 360mc. The article describes individual development stages from the concept to production itself including a final visualization of the prototype model of an 11-blade axial fan.

Keywords: Axial Fan, Additive Technology, Rapid Prototyping, Fused Deposition Modeling, 3D Printer

\section{Acknowledgement}

The research was supported and co-financed from the project called „TA CR GAMA PP1 TG01010054 - VUT SANCE“.

\section{References}

[1] ${ }^{\circ} 2015$ VYTOZ-EKO spol. s.r.o. Ventilacni turbiny ZLT. All rights reserved. [Online]. [Seen 27 $7^{\text {th }}$ May 2015]. Available at: http://www.vytozeko.cz/ventilacni-turbiny-zlt/.

[2] ${ }^{\circ} 2015$ H-tech group s.r.o. Ventilacni turbiny a vetraci ventilatory. All rights reserved. [Online]. [Seen $27^{\text {th }}$ May 2015]. Available at: http://www.vetrani.com/index.php/cs/vv-ventilacni-turbiny.

[3] ${ }^{\circ} 2015$ Haze s r.o. Lomanco ventilacni turbina. [Online]. [Seen 27 $7^{\text {th }}$ May 2015]. Available at: http://www.haze.cz/stresni-vetraci-systemy/lomanco-ventilacni-turbina/.

[4] 2015 MOBILAND TRADE s.r.o., Edmonds. Neco o ventilaci. [Online]. [Seen 27 $7^{\text {th }}$ May 2015]. Available at: http://www.ventilacniturbiny.cz/neco-o-ventilaci.

[5] ONDRA, M. (2015). Vyvoj a regulace ventilacnich turbin. Brno. Master Thesis. Brno University of Technology, Faculty of Mechanical Engineering, Institute of Automotive Engineering. 84 p., 15 appendices. Advisor doc. Ing. Jiri Malasek, Ph.D.

[6] Edmonds. In: Hurricane Brochure. [Online]. [Seen $18^{\text {th }}$ April 2015]. Available at: http://www.edmonds.com.au/Edmonds/UploadedFiles/40/400d36aa-8717-47d7-adc8-b5cf04f32686.pdf.

[7] Edmonds. In: Technicke specifikace windmaster, superwhirly. [Online]. [Seen 18 ${ }^{\text {th }}$ May 2015]. Available at: http://www.ventilacniturbiny.cz/technicke-dokumenty/superwhirly/superwhirly-tech-specifikace.pdf.

[8] Edmonds. In: Technicke specifikace supavent, turbobeam. [Online]. [Seen $18^{\text {th }}$ May 2015]. Available at: http://www.ventilacniturbiny.cz/technicke-dokumenty/turbobeam/turbobeam-tech-specifikace.pdf.

[9] Edmonds. In: Technicke specifikace turbo ventura, sewer vent. [Online]. [Seen 18 $8^{\text {th }}$ May 2015]. Available at: http://www.ventilacniturbiny.cz/technicke-dokumenty/sewervent/sewervent-tech-specifikace.pdf.

[10] Insight 10.2 ${ }^{\circledR}$. ${ }^{\circ} 2015$ Stratasys Ltd. All rights reserved. [Online]. [Seen. $21^{\text {st }}$ September 2015]. Available at: http://www.dimensionprinting.com/applications/rapid-prototyping.aspx.

[11]CHUA, C. K., LEONG, K. F., LIM, C. S. (2010). Rapid Prototyping: Principles and Applications. $3^{\text {rd }}$ Ed. New Jersey: World Scientific, 512 p. ISBN 978-981-277-897-0.

[12] GEBHARDT, A. (2003). Rapid Prototyping. CARL HANSER VERLAG, 377 p. ISBN 3-446-21259-0. 
[13]SEDLAK, J., PTACKOVA, M., NEJEDLY, J., MADAJ, M., DVORACEK, J., ZOUHAR, J., CHARVAT, O., PISKA, M. (2013). Material Analysis of Titanium Alloy Produced by Direct Metal Laser Sintering. International Journal of Metalcasting, Vol. 7, No. 2, p. 43-50. ISSN 1939-5981.

[14]MARCINCIN, J. N., JANAK, M., MARCINCINOVA, L. N. (2012). Increasing of Product Quality Produced by Rapid Prototyping Technology. Manufacturing Technology, June, Vol. 12, No. 12, p. 71-75. ISSN 1213-2489.

[15]SEDLAK, J., RICAN, D., PISKA, M. (2015). Study of Materials Produced by Powder Metallurgy Using Classical and Modern Additive Laser Technology. Procedia Engineering, Vol. 2015, No. 1, p. 1232-1241. ISSN $1877-$ 7058 .

[16]SEDLAK, J., SEKERKA, V., SLANY, M., KOURIL, K., ZEMCIK, O., CHLADIL, J., ROZKOSNY, L. (2015). Production of Prototype Parts Using Direct Metal Laser Sintering Technology. Acta Polytechnica, 2015, Vol. 55, No. 4, p. 260-266. ISSN 1210-2709.

[17]SEDLAK, J., SLANY, M., FIALA, Z., JAROS, A. (2015). Production Method of Implant Prototype of KneeJoint Femoral Component. Manufacturing Technology, 2015, Vol. 15, No. 2, p. 195-204. ISSN 1213-2489.

[18]SHIGLEY, J. E., MISCHKE, Ch. R., BUDYNAS, R. G. (2010). Konstruovani strojnich soucasti. Brno: VUTIUM, 1186 p. ISBN 978-80-214-2629-0.

[19]LEINVEBER, J., VAVRA, P. (2011). Strojnicke tabulky. $5^{\text {th }}$ Ed. Uvaly: ALBRA, 927 p. ISBN 978-80-7361$081-4$. 